\title{
IUFOST2006/745 Characterisation of Fruits by Multivariate Image Analysis
}

\author{
P. Guillermin ${ }^{\mathrm{a}}$, D. Bertrand ${ }^{\mathrm{b}}$, S. Chevallier ${ }^{\mathrm{b}}$ and A. Kohler \\ ${ }^{a}$ Institut National d'Horticulture, 2, rue le Nôtre, 49045 Angers Cedex 01, France \\ ${ }^{\mathrm{b}}$ ENITIAA, Nantes, La Géraudière BP 82225, 44322 Nantes cedex 03, France \\ ${ }^{\mathrm{c}}$ Centre for Biospectroscopy and Data Modelling, Matforsk, Norwegian Food Research Institute, 1430 Ås, \\ Norway \\ pascale.guillermin@inh.fr
}

The purpose of the present study was to design and test a simple imaging system making it possible to characterise large food samples (from about $1 \mathrm{~cm}$ to $20 \mathrm{~cm}$ ). The system has been used for the characterisation of the heterogeneity of apples and the browning of the fruit after cutting. The imaging system called "multiway imager" (MUWI) includes a 12-bit CCD cooled RGB camera and an illuminating system formed by bunches of easily available LEDs working at 8 different wavelengths ranging from $400 \mathrm{~nm}$ to $950 \mathrm{~nm}$. Each bunch included 12 LEDs distributed at the 4 points of a square in order to light up the sample evenly. The system makes it possible to control the photo accumulation from a few milliseconds to about 20 seconds. For each of the 8 LED illuminations, an RGB image was acquired, giving 3 channels associated with Red, Green and Blue. An object is thus characterised by $8 \times 3=24$ image-plans. The integration time was adjusted separately for each illumination condition. In a previous study the capability of the system to discriminate raw materials has been investigated. In the present work, apples belonging to 4 cultivars (Caméo, Granny, Golden, Gala) were studied. The fruits were sectioned and placed in the imaging system. The multivariate images of the whole equatorial section of the fruits were acquired, either immediately after the cutting of the fruits or after 15 minutes. Each multivariate image formed an image-cube with 524 rows and 702 columns, corresponding to the spatial dimensions, and 24 image-plans associated with the illumination conditions. For each pixel of each plan, the intensity of light was expressed as $\log (\mathrm{I} 0 / \mathrm{I})$, with I: the grey-level value of the pixel, and I0: the intensity measured with a white reference standard, at the same location. The images were thresholded in order to isolate the images of the fruit section from the background. A mathematical morphology operation was then applied in order to compute the distance of every pixel from the external boundary of the silhouette of the fruits. The nature of the cultivar, the time of exposure after cutting the fruit and the distance from the boundary were studied using factorial methods. The methods make it possible to characterise the spatial heterogeneity of the fruits. Every studied effect was statistically significant. The possibility of using the system for studying food products other than fruits will be discussed. 